\title{
Methodological Paper: Employing Neural Network Methods to Label Sleep EEG Micro-Arousals in Obstructive Sleep Apnea Syndrome
}

\author{
Mohammad Nami ${ }^{1,2^{*}}\left(\mathbb{D}\right.$, Samrad Mehrabi ${ }^{2,3}$, Sabri Derman ${ }^{4} \mathbb{C}$
}

1. Department of Neuroscience, School of Advanced Medical Sciences and Technologies, Shiraz University of Medical Sciences, Shiraz, Iran. 2. Sleep Disorders Laboratory, Namazi Hospital, Shiraz University of Medical Sciences, Shiraz, Iran.

3. Department of Internal Medicine, School of Medicine, Shiraz University of Medical Sciences, Shiraz, Iran.

4. Sleep Disorders Unit, American Hospital, Koc Foundation, Istanbul, Turkey.

\section{Article info:}

Received: 24 Jul 2017

Accepted: 29 Oct 2017

Keywords:

Sleep apnea, EEG,

Microstructure, Arousals,

Neural network methods
Cittation: Nami M, Mehrabi S, Derman S. Employing Neural Network Methods to Label Sleep EEG Micro-Arousals in Obstructive Sleep Apnea Syndrome. JAMSAT. 2017; 3(4):221-226. https://doi.org/10.32598/jamsat.3.4.221

https://doi.org/10.32598/jamsat.3.4.221

\begin{abstract}
Well-designed studies are essential to screen suspected cases of Obstructive Sleep Apnea Syndrome (OSAS) using the widely-referenced questionnaires and then to confirm the diagnosis by means of full Polysomnography (PSG), and finally to assess various variables of treatment efficacy and safety. Defining the severity index of OSAS based on the ApneaHypopnea Index (AHI), sleep marco- and micro-structural features (i.e. hypnogram and cyclic alternating patterns or CAPs), and neurocognitive functions would help better explain the treatment outcome. Using the neural network models on sleep data in OSAS sufferers is potentially expected to help the above goals. Determination of neurocognitive impairments in OSAS subjects in relation with disease severity indices and subsequent changes in microstructural changes (i.e. CAPs) in sleep Electroencephalography (EEG), would therefore be useful in defining individualized care and cognitive rehabilitation plans. The present methodology paper has attempted to address the above hypothesis in a clinical population from a hospital-based sleep disorders laboratory.
\end{abstract}

\section{Introduction}

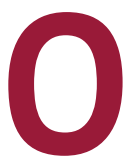

bstructive Sleep Apnea Syndrome (OSAS) is a clinically important and relatively prevalent sleep disorder [1]. Epidemiological investigations have reported the OSAS prevalence between $3 \%$ and $28 \%$ suggesting a wide range of dissimilarity across studies [2]. The severity of this clini- cal condition is defined based on the mean number of sleep apnea and hypopnea events per hour, known as the Apnea-Hypopnea Index (AHI) [3]. The collective nighttime consequences of OSAS include periodic nocturnal oxygen desaturations, increased blood pressure, and arousal instability during sleep. In addition, OSAS is associated with cardiovascular consequences, including but not limited to hypertension, coronary heart disease, and stroke [4].

* Corresponding Author:

Mohammad Nami, MD, PhD

Address: Department of Neuroscience, School of Advanced Medical Sciences and Technologies, Shiraz University of Medical Sciences, Shiraz, Iran. Tel: +98 (71) 32305471

E-mail: torabinami@sums.ac.ir 
From the neurological and psychiatric viewpoints, OSAS is known to contribute to affective, cognitive, and autonomic nervous system changes [5]. Based on the existing empirical data, neurocognitive deficits tend to progress in OSAS cases who are not timely treated [6]. While the effect of OSAS on visual and verbal memory functions is variable [7], cognitive and executive functions, including reasoning, comprehension, and learning are among the domains commonly affected [5].

In some instances, despite standard treatments including continuous positive airway pressure therapy, overactivity of sympathetic flow as well as cognitive and mood changes continue to persist. This indicates that dysregulated neural mechanisms may be responsible for at least some of the characteristics observed in OSAS [8]. Such patients may continue to complain from irritability, impaired attention, and vigilance as well as emotional instability or mood swings. In reality, even the patients with less intense sleep-disordered breathing (including patients with upper airway resistance syndrome-UARS) are potentially prone to neurocognitive impairments [9].

Since OSAS is shown to significantly interfere with duration, depth and continuity of sleep, it negatively affects the sleep restorative function. This effect not only reflects in sleep Electroencephalography (EEG), but also in autonomic and behavioral functions. In addition, OSAS is known to affect Cyclic Alternating Patterns (CAPs) in sleep EEG as a marker of sleep arousal instability [9]. Taking the above evidence into consideration, some studies have attempted to pursue: (a) Assessment of arousal instability by means of CAP parameters in OSAS patients compared with age-matched controls; (b) Investigation of the reciprocal interactions between CAPs and neurocognitive performance (attention, memory, learning and executive functions) during wakefulness; and (c) Using the neural networks with sleep data in OSAS sufferers [9-12].

The density of micro-arousals in sleep EEG is known to be inversely correlated with neurocognitive functions in cases complaining from unrefreshing sleep. This correlation has less been examined in cases with OSAS [10-12]. As such, the abundance of sleep apnea events and subsequent cortical excitability reflected in CAPs may result in arousal instability during sleep $[10,13]$. In other words, inadequately treated OSAS is potentially linked to arousal instability during sleep and continues to negatively affect the brain especially in chronic state [5]. This brief report highlights some methodological perspectives upon investigating the relation between arousal instability, CAPs and neurocognitive performance in a population of OSAS patients evaluated at our hospital-based sleep disorders laboratory.

\section{Materials and Methods}

Based on the evidence and screening outcome as per the cross-sectional and prospective investigations initial screening was performed to isolate high-risk patients for OSAS. Related questionnaires, including Addenbrooke's Cognitive Examination (ACE) and STOP-Bang were employed to shortlist suspected OSAS cases for polysomnographic evaluations at out full-night PSG setting. Primary sampling was done in a consecutive manner from the patients who referred to sleep disorders clinic from 2015 to 2017.

To confirm the diagnosis in clinically-suspected OSAS subjects in high-risk population for OSAS, based on the sample size calculation (Fleiss with continuity correction), the study was conducted on 28 cases of mild to moderate OSAS) with the apnea hypopnea index of 15 or more, confirmed upon polysomnography) as well as age- and sex-matched healthy control subjects who were enrolled for overnight polysomnographic assessment. Assessments were done at our hospitalbased sleep disorders laboratory.

During the Polysomnography (PSG) test, the following data, including EEG (F3-A2, F4-A1, C3-A2, C4-A1, O1-A2), electrooculograhy (right and left), electromyography (submental), electromyography )right and left anterior tibialis, (breathing effort) chest and abdomen), air intake (mouth/nose air flow), snoring sounds, oxygen saturation, plethysmography, electrocardiography, heart rate and sleeping position were recorded in a software and later scored and analyzed by a sleep specialist. The PSG data were analyzed and scored by a polysomnography technologist with clinical expertise in sleep medicine using the latest scoring rules from the American Academy of Sleep Medicine (AASM). Additionally, manual scoring for CAPs was done by a PSG/sleep EEG expert.

Patients with confirmed diagnosis of OSAS (based on PSG) underwent neurocognitive evaluations using the ACE tool to get their attention, memory, learning and executive function capacity examined. The same set of data were obtained from a group of sex- and age-matched control subjects and correlation analysis was done between control and OSAS group. This procedure was done and completed over a period of 16 months [9]. 

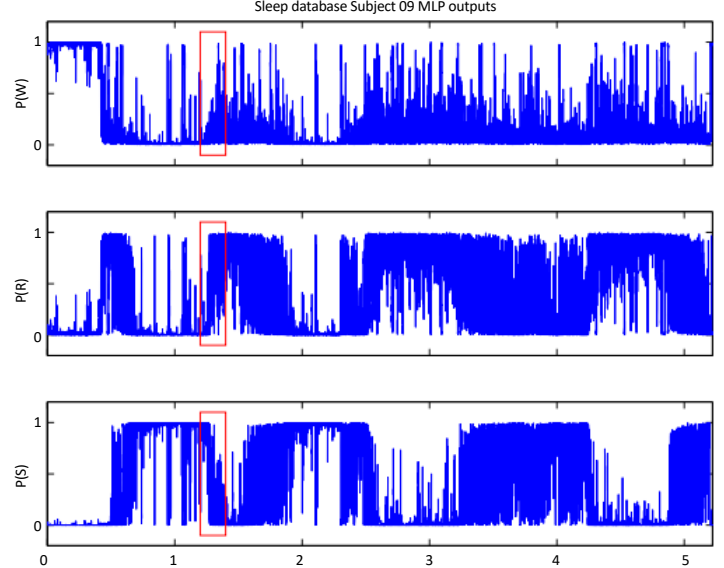
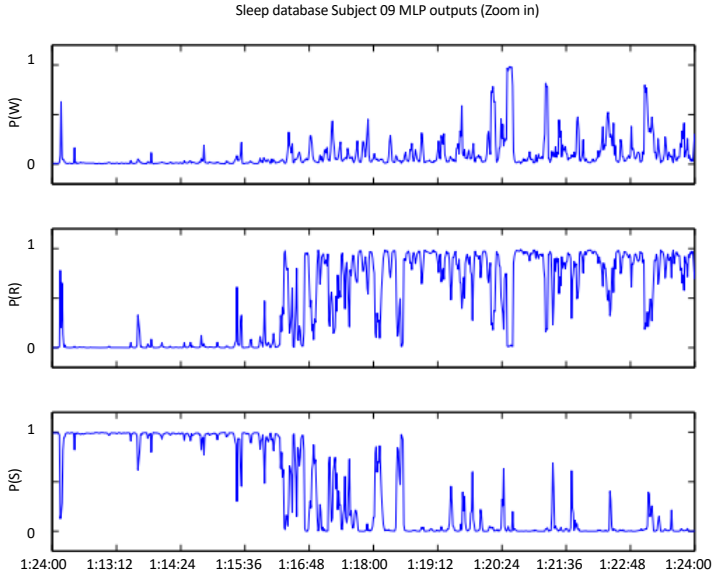

JAMSAT

Figure 1. MLP outputs, $P(W), P(R)$ and $P(S)$ for subject 9's all-night record, showing a 12-minute segment detailed

All-night sleep EEG recording from 28 cases using the American Academy of Sleep Medicine (AASM) electrode montage were independently scored for staging and micro-arousals by two human experts on a 30-second basis. The optimal Multilayer Perceptron (MLP) network was used to analyze the all-night EEG record of a subject from the normal sleep and OSAS database. The time courses of two of the three MLP outputs were combined to give a measure of sleep depth $[P(W)-P(S)]$, which shows a high correlation with the hypnogram generated by a human expert, suggesting that the MLP is able to interpolate between classes for sleep stages 2 and 3. The sleep EEG of OSAS subjects was analyzed using the optimal MLP. The time courses of the $[P(W)-P(S)]$ output show severe disruption in the sleep. A method for automated micro-arousal detection using thresholding of the $[P(W)-P(S)]$ output was introduced aiming to analyze the sleep macro- and micro-structure-related data for each subject and to associate among the severity index of OSAS based on AHI, sleep marco- and micro-

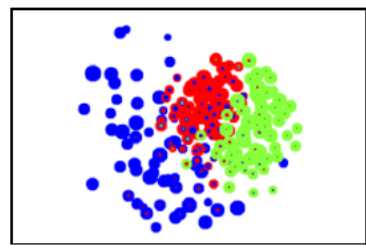

(a) Classes W, Rand S

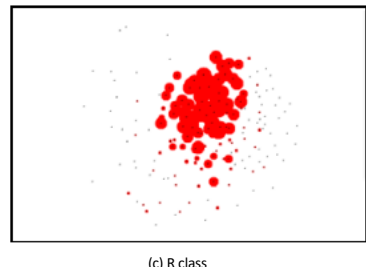

structural features (i.e. hypnogram and CAPs), and neurocognitive functions (based on ACE) [9].

The differences between OSAS and control groups was assessed using the ANCOVA, with age and AHI as covariates $(P<0.05)$, corrected for multiple comparisons using false discovery rate. Apart from $\mathrm{AHI}$, the correlation between mean $\mathrm{SaO}_{2}$ and minimum $\mathrm{SaO}_{2}$ (revealed by continuous pulse oximetry during PSG) with cognitive functions and CAP distribution was assessed during statistical analyses. In addition, sLORETA was used to source-localize phases A1 and B of CAPs.

\section{Results}

The output of the automated scores was post-processed to follow AASM rules for micro-arousal scoring as closely as possible. Sensitivity, positive predictive accuracy, and correlation were used to evaluate the performance of the automated detection system with respect to the human expert scores. The correlation measure
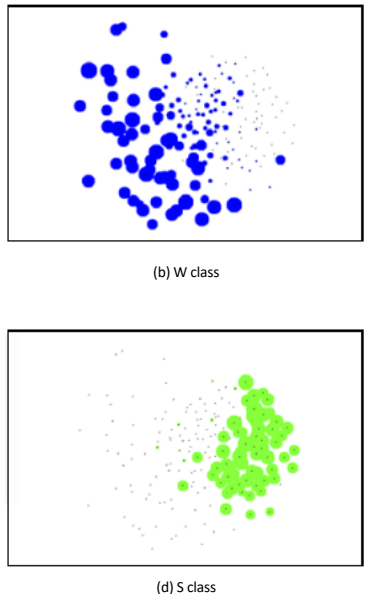

(d) 5 class 

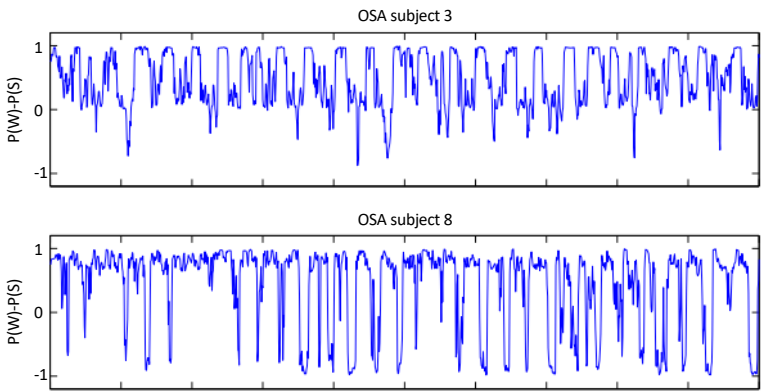

Normal sleep subject

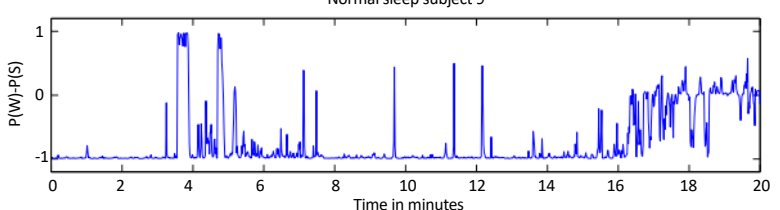

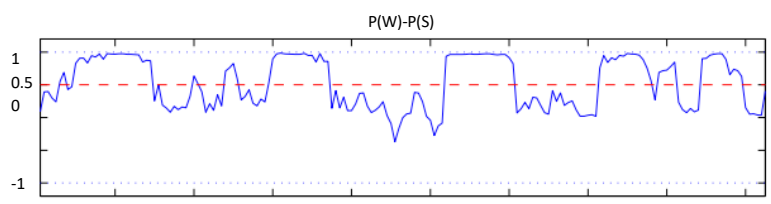

After thresholding

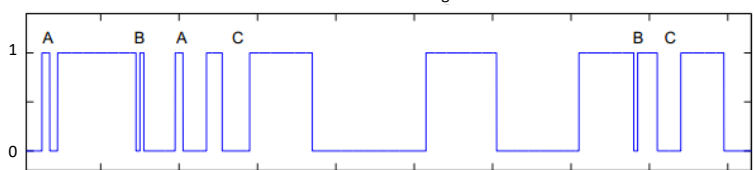

Including 3s and 10S ASDA Criteria

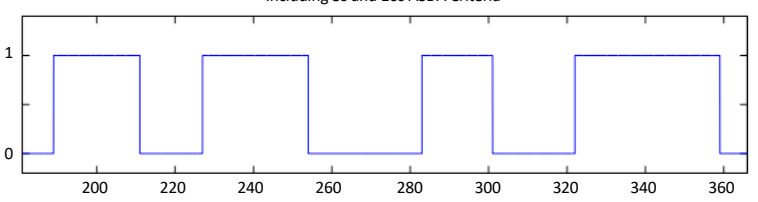

JAMSAT

Figure 3. $\mu$-Arousal detection procedure; Upper trace: $[\mathrm{P}(\mathrm{W})-\mathrm{P}(\mathrm{S})]$ and a 0.5 threshold; Middle trace: Thresholding result; Lower trace: $\mu$-Arousal automatic score with AASM timing criteria

was used to choose the optimal threshold value per subject, and two methods for setting the threshold, one of them subject-adaptive, were applied retrospectively.

The results for 28 moderate to severe OSAS subjects (mean[SD] AHI of 42[11]) show a high correlation (greater than 0.8) value. The neural network, trained with normal sleep data appears to be capable of following the abrupt transitions in the sleep EEG of OSAS patients (Figures 1, 2, 3, and 4).

\section{Conclusion}

The methods introduced for automated micro-arousal detection were able to identify a high percentage of the events scored by the human experts, giving the beginning and the end times for the micro-arousal with relatively high accuracy (as measured by a simple correlation index) for OSAS subjects in our database. The study of the subjects with low correlation levels in the automated micro-arousal detection showed different changes in the EEG frequency content prior to and during the micro-arousal. The 3-second analysis window has yielded better results in terms of MLP performance and in the sensitivity and correlation of the micro-arousal detection.

\section{Ethical Considerations}

\section{Compliance with ethical guidelines}

All data acquisition processes followed the ethical guidelines laid down by the ethics committee at Shiraz University of Medical Sciences and received the related
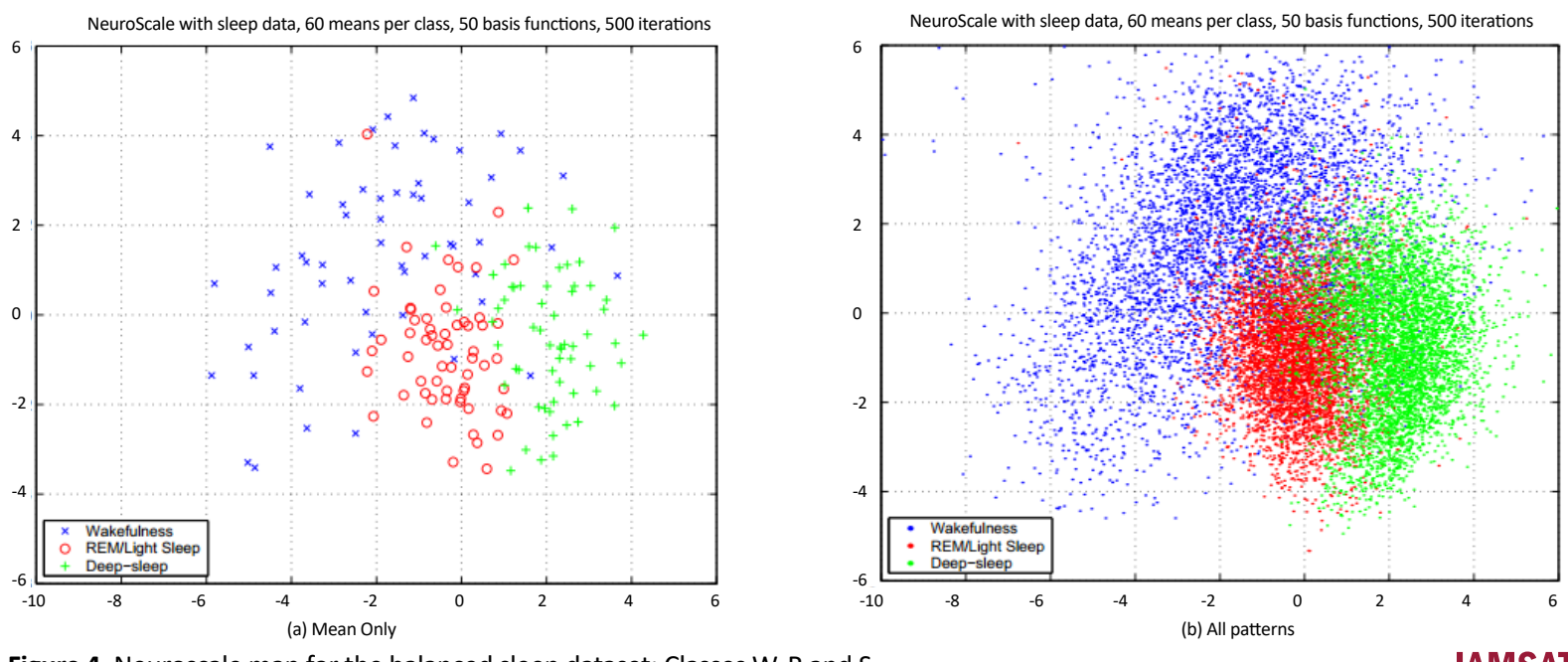

Figure 4. Neuroscale map for the balanced sleep dataset; Classes W, R and S 
approval by the institutional review board under the approval No. 9088-74-01-93.

\section{Funding}

The present article is a part of the project 9088-74-0193 supported by the Iranian Cognitive Science and Technologies council, IR Iran.

\section{Conflict of interest}

Authors declared no conflict of interest.
[10] Milioli G, Bosi M, Grassi A, Riccardi S, Terzano MG, Cortelli P, et al. Can sleep microstructure improve diagnosis of OSAS? Integrative information from CAP parameters. Archives Italiennes de Biologie. 2015; 153(2-3):194-203. [PMID]

[11] Smerieri A, Parrino L, Agosti M, Ferri R, Terzano MG. Cyclic alternating pattern sequences and non-cyclic alternating pattern periods in human sleep. Clinical Neurophysiology. 2007; 118(10):2305 13. [DOI:10.1016/j.clinph.2007.07.001] [PMID]

[12] Thomas RJ, Terzano MG, Parrino L, Weiss JW. Obstructive sleep-disordered breathing with a dominant cyclic alternating pattern: A recognizable polysomnographic variant with practical clinical implications. Sleep. 2004; 27(2):229-34. [DOI:10.1093/ sleep/27.2.229] [PMID]

[13] Terzano MG, Parrino L, Boselli M, Spaggiari MC, Di Giovann G. Polysomnographic analysis of arousal responses in obstructive sleep apnea syndrome by means of the cyclic alternating pattern. Journal of Clinical Neurophysiology. 1996; 13(2):145-55. [DOI:10.1097/00004691-199603000-00005] [PMID]

\section{References}

[1] Baldi I, Gulati A, Lorenzoni G, Natarajan K, Ballali S, Kameswaran $M$, et al. Public health implications of obstructive sleep apnea burden. Indian Journal of Pediatrics. 2014; 81(1):55-62. [DOI:10.1007/ s12098-014-1539-8] [PMID]

[2] Young T, Palta M, Dempsey J, Peppard PE, Nieto FJ, Hla KM. Burden of sleep apnea: Rationale, design, and major findings of the Wisconsin Sleep Cohort study. World Medical Association. 2009; 108(5):246-9. [PMID] [PMCID]

[3] Madbouly EM, Nadeem R, Nida M, Molnar J, Aggarwal S, Loomba $R$. The role of severity of obstructive sleep apnea measured by apnea-hypopnea index in predicting compliance with pressure therapy: A meta-analysis. American Journal of Therapeutics. 2014; 21(4):260-4. [DOI:10.1097/MJT.0b013e318249a09d] [PMID]

[4] Hsieh SW, Lai CL, Liu CK, Hsieh CF, Hsu CY. Obstructive sleep apnea linked to wake-up strokes. Journal of Neurology. 2012; 259(7):1433-9. [DOI:10.1007/s00415-011-6370-9] [PMID]

[5] Torabi-Nami M, Mehrabi S, Borhani-Haghighi A, Derman S. Withstanding the obstructive sleep apnea syndrome at the expense of arousal instability, altered cerebral autoregulation and neurocognitive decline. Journal of Integrative Neuroscience. 2015; 14(2):169-93. [DOI:10.1142/S0219635215500144] [PMID]

[6] Gelir E, Basaran C, Bayrak S, Yagcioglu S, Budak MT, Firat H, et al. Electrophysiological assessment of the effects of obstructive sleep apnea on cognition. PLoS One. 2014; 9(2):e90647. [DOI:10.1371/ journal.pone.0090647] [PMID] [PMCID]

[7] Beebe DW, Groesz L, Wells C, Nichols A, McGee K. The neuropsychological effects of obstructive sleep apnea: A meta-analysis of norm-referenced and case-controlled data. Sleep. 2003; 26(3):298-307. [DOI:10.1093/sleep/26.3.298] [PMID]

[8] Canessa N, Castronovo V, Cappa SF, Aloia MS, Marelli S, Falini A et al. Obstructive sleep apnea: Brain structural changes and neurocognitive function before and after treatment. American Journal of Respiratory and Critical Care Medicine. 2011; 183(10):141926. [DOI:10.1164/rccm.201005-06930C] [PMID]

[9] Karimzadeh F, Nami M, Boostani R. Sleep microstructure dynamics and neurocognitive performance in obstructive sleep apnea syndrome patients. Journal of Integrative Neuroscience. 2017; 16(2):127-42. [DOI:10.3233/JIN-170004] [PMID] 
